SECTION 2. Applied mathematics. Mathematical modeling.

Marina Yurievna Paklyachenko

Post-graduate cadet of the Information Security chair Voronezh Institute of the Ministry of the Interior of Russia marina_lion@mail.ru

\title{
COMPARATIVE ANALYSIS OF DATA COMMUNICATIONS SYSTEMS AND TRANSPORT SYSTEMS MODELING : MODELS OF THE CORRESPONDENCE MATRIX COMPOUND.
}

\begin{abstract}
The article constitutes a basic review of theoretical statements dealing with the aspects of compound multi-channel system mathematical modeling. The work treats and summarizes the knowledge on the describing the quantitative and dynamic characteristics (correspondence matrix) of the referred systems. The examples of the matrix compound in the gravitational, entropy, intermediate capabilities and rival centers models are given.

Key words: model, multi-information systems, correspondence matrix, parcel, channel, stream.

\section{СРАВНИТЕЛЬНЫЙ АНАЛИЗ МОДЕЛЕЙ СИСТЕМ ПЕРЕДАЧИ ДАННЫХ И ТРАНСПОРТНЫХ СИСТЕМ: МОДЕЛИ ФОРМИРОВАНИЯ МАТРИЦЫ КОРРЕСПОНДЕНЦИЙ.}

Аннотация: В работе рассматриваются основные теоретические положения, касающиеся вопросов математического моделирования сложных многоканальных систем. Статья анализирует и обобщает способы описания количественных и динамических характеристик (составление матрии корреспонденций) рассматриваемых систем. Приводятся примеры составления матрии в рамках гравитационной и энтропийной моделей, моделей конкурирующих иентров $и$ промежуточных возможностей.

Ключевые слова: модель, многоканальные информачионные системы, матрица корреспонденций, посылка, канал, поток.

\section{Введение}

Математические модели, применяемые для анализа транспортных и информационных сетей обладают несомненным сходством, обусловленным понятийной и функциональной близостью их элементов. Ввиду больно количества типов и модификаций моделируемых объектов их формализованные описания довольно разнообразны. Это обусловлено особенностями поставленных задач и математическим аппаратом, применяемым для их решения задач изучения поведения магистральных или же информационных процессов.

Условно можно разделить всю совокупность моделей на 3 базисные группы: прогнозные (например, прогноз загрузки транспортной сети и сети передачи информации, состоящий в усреднении характеристик движения: объемов межмагистральных или межканальных передвижений, интенсивности потока, перераспределения потоков автомобилей и пассажиров или передаваемых данных по направлениям движения, трафика), имитационные (например, динамика транспортного или информационного потока, описывающая развитие процесса его движения и перераспределения во времени, скорость, задержку, длины и динамику образования 
"очередей, заторов") и оптимизационные (задачи оптимизации маршрутов пассажирских и грузовых перевозок, пакетов передачи данных, выработка оптимальной конфигурации сети) [3, с. $124 ; 8$, с. 55].

Актуальными на данный момент выступают имитационные модели, направленные на разработку систем автоматизированного управления движением, где транспортный или информационный поток рассматривается как физическое явления со сложными нетривиальными свойствами (например, потеря устойчивости для транспортной системы, или непреднамеренное воздействие деструктивного характера на данные в сети передачи инфомрации, явление самоорганизации и коллективного поведения транспорта, или возникновение фракций контрагентов - элементарных процессов обработки информации, функционирующих ненадлежащим образом) [7, с. 47].

\section{Основные определения}

Целесообразно введение определенных дефиниций.

Межрайонная корреспонденция (межканальная корреспонденция) - общий объем передвижений из одного района/канала/узла (прибытия/отправления) в другой.

Обобщенная цена пути - критерий (сумма различных факторов, главный из которых - время), выражаемый математически, на основании которого оцениваются альтернативные пути и способы передвижения. Чем выше цена, тем ниже привлекательность пути.

Матрица корреспонденций - количественная характеристика структурности транспортной/информационной системы, элементы которой описывают объемы передвижений между каждой парой условных районов/каналов/магистралей $[1$, с. $78 ; 2$, c. $113 ; 9$, с. 3].

При составлении матрицы корреспонденций в рамках решаемой задачи, учитывая свойство изоморфности системы [4, с. 55], обобщенная цена будет являться числовым выражением транспортного расстояния.

Реальные транспортные потоки стремятся обрести равновесное (балансное) состояние при прямой зависимости цены путей от загрузки системы и обратной, при которой загруженность системы будет определяться стоимостью путей.

Актуальными для реализации поиска равновесного состояния транспортных / информационных сетей являются итерационные процессы [5, с. 118], состоящие из 4х взаимосвязанных и взаимозависимых подэтапов, обуславливающих необходимость решения задачи методами последовательных приближений итерационного цикла:

-оценка общих объемов прибытия и отправления из каждого района/канала/пути;

-расщепление по способам передвижений / учет межканальных связей;

-определение матриц, характеризующих объем передвижений с учетом взаимосвязей районов / каналов (матрицы корреспонденции);

-распределение корреспонденций по сети (определение путей/ характеристик потока).

\section{Составление матрицы корреспонденций}

При составлении матрицы основополагающей, исходной информацией будет значение общих объемов прибытия и отправления транспортных единиц (данных) в каждом районе/канале/узле.

Оценка объема может дифференцироваться по разным группам, учитывая и пространственное размещение потокопораждающих объектов и подвижность 
населения/пропускную способность применяемых каналов связи, т.е средним количеством поездок/пересылок. Зачастую, такую оценку, предшествующую моделированию, получают опытным путем (социально-экономические данные / эмпирические статистические данные, определяемые техническими и эксплуатационными характеристиками применяемого оборудования).

Вопрос распределения затрагивает два этапа: модальный выбор - выбор способа передвижения, реализуемый на стадии расчета корреспонденции; критериальный выбор - выбор конкретного пути передвижения на основе некоторого критерия оценки тракта, реализуемый как результат распределения.

\section{Гравитационная модель}

Основная идея гравитационной модели заключается в следующем: корреспонденция из одного района/ узла системы в другой район/узел/канал пропорциональная общему объему отправления и общему объему прибытия из одного районного/канального/узлового центра в другой, и некоторой функции зависящей от транспортного расстояния (степени близости/межканального взаимодействия) между описываемыми центрами [6, с. 59].

Модель можно представить следующими выражениями, определяющими корреспонденцию пересылки и условие балансного состояния:

$$
\begin{array}{r}
M_{i j}=A_{i} B_{j} C_{i} D_{j} T\left(r_{i j}\right) \\
\sum_{i \in R} A_{i}=\sum_{j \in R} B_{j},
\end{array}
$$

где $\mathrm{M}_{\mathrm{ij}}$ - корреспонденция из района/канала i в $\mathrm{j}$,

$\mathrm{T}\left(\mathrm{r}_{\mathrm{ij}}\right)$ - функция тяготения, зависящая от транспортного расстояния $\mathrm{r}_{\mathrm{ij}}$,

$A_{i}$ - объем отправления / передачи в район/канал,

$\mathrm{B}_{\mathrm{j}}$ - объем прибытия / получения в район/ в канале.

В зависимости от типа корреспонденций объемы $\mathrm{A}_{\mathrm{i}}$ и $\mathrm{B}_{\mathrm{j}}$ измеряются в автомобилях, пассажирах / битах, пакетах, других удобных транспортных единицах и определяются из условий:

$$
\begin{aligned}
& \sum_{j \in R} M_{i j}=A_{i}, \quad i \in R, \\
& \sum_{i \in R} M_{i j}=B_{j}, \quad j \in R, \\
& M_{i j} \geq 0, \quad i, j \in R .
\end{aligned}
$$

Касательно уравнения баланса системы, возможна корректировка исходных данных путем их умножения на поправочные коэффициенты для выполнения условия баланса.

Функция $\mathrm{T}\left(\mathrm{r}_{\mathrm{ij}}\right)$ является первостепенным фактором, характеризующим распределение передвижений по дальности. Ее выбор осуществляется при калибровке модели на основе сопостановления выходных дальностей с эмпирическими показателями.

Релевантна следующая аппроксимация:

$$
\mathrm{T}\left(\mathrm{r}_{\mathrm{ij}}\right)=\exp \left(-\alpha \mathrm{r}^{\beta}\right), \alpha \geq 0, \beta \geq 0 .
$$

Вычисление $\mathrm{M}_{\mathrm{ij}}$ сводится к расчету коэффициентов $\mathrm{C}_{\mathrm{i}}$ и $\mathrm{D}_{\mathrm{j}} \mathrm{c}$ применением итерационных методов решения уравнений (1) (2) .

\section{Энтропийная модель}


Данная модель, разработанная Вильсоном, нашла широкое применение в области решения транспортных задач [10, с. 256].

Концепция модели основывается на вероятностном описании возможных состояний поведения потоков. Вводится определение состояния с наибольшим статистическим весом - реального состояния. Преимущество использования данной энтропийной характеристики объясняется возможным отсутствием существования конечного и нормированного распределения вероятностей.

Энтропия системы определяется следующим образом:

$$
\mathrm{H}(\mathrm{m})=\sum_{\mathrm{i}, \mathrm{j}} \mathrm{m}_{\mathrm{ij}} \ln \frac{\mathrm{m}_{\mathrm{ij}}}{\gamma_{\mathrm{ij}}}, \mathrm{m}=\left\{\mathrm{m}_{\mathrm{ij}} \mid \mathrm{i}, \mathrm{j} \in \mathrm{R}\right\},
$$

$\mathrm{m}_{\mathrm{ij}}$ - числа заполнения состояний, т.е количество элементов системы.

$\gamma_{\mathrm{ij}}$ - более вероятные значения $\mathrm{m}_{\mathrm{ij}}$.

Наиболее вероятные корреспонденции $\mathrm{M}_{\mathrm{ij}}$ определяются как максимизация энтропии при некоторой системе ограничений на числа заполнения состояний.

В отсутствии ограничений:

$$
\mathrm{M}_{\mathrm{ij}}=\gamma_{\mathrm{ij}} \text {. }
$$

Ограничениями могут выражаться через индивидуальные характеристики системы (например, затратные) и транспортные требования (ограничения баланса отправок и получений). Учитывая их наличие, преобразуем выражение модели:

$$
\begin{gathered}
M=\underset{j \in R}{\operatorname{argmax}(H(m)),} m=\left\{m_{i j} \mid i, j \in R\right\} \\
\sum_{i \in R} m_{i j}=A_{i}, \quad i \in R, \\
m_{i j} \geq 0, \quad i, j \in R, \\
g(m)_{l}=0, \quad l=1 \cdots L, \\
h(m)_{p} \leq 0, \quad p=1 \cdots P .
\end{gathered}
$$

Если в рамках рассматриваемой задачи более вероятные значения корреспонденций равны $\left(\gamma_{\mathrm{ij}}=\mathrm{const}\right)$, и имеются балансные характеристические ограничения, то ее решение совпадает с гравитационной моделью:

$$
\mathrm{T}(\mathrm{r})=\exp \left(-\alpha \mathrm{r}_{\mathrm{ij}}\right) \text {, }
$$

$\alpha$ - множитель Лагранжа задачи оптимизации, значение которого определяется в ходе решения задачи.

\section{Модель конкурирующих центров}

Основным аспектом данной модели является факт привлекательности определенного района/канала для посещения/отправки, что определяется его расположением по отношению к другим районам/каналам/узлам.

Рассматриваемая модель представляет собой расширенную гравитационную модель с добавлением дополнительных выражений.

Вводится новая характеристика, позволяющая моделировать алгромерационные/конурбационнные процессы в структуре системы - индекс посещаемости/коэффициент востребованности района/канала, выражаемый следующим образом:

$$
\mathrm{V}_{\mathrm{ij}}=\sum_{\mathrm{k} \in \mathrm{R}, \mathrm{k} \neq \mathrm{i}, \mathrm{j}} \frac{\mathrm{B}_{\mathrm{k}}}{\mathrm{r}_{\mathrm{k}_{\mathrm{j}}}}
$$

Величина коэффициента определяется степенью близости к рассматриваемому району/каналу альтернативных направлений. 


\section{Модель промежуточных возможностей}

Была предложена Стауффером в рамках транспортного распределения потоков и заключается в следующем [11, с. 845]. Объем корреспонденции между двумя центрами определяется не расстояниями между ними, а количеством и емкостью альтернативных центров прибытия на пути, т.е количеством альтернативных возможностей прохождения:

$$
\mathrm{m}_{\mathrm{n}}=\mathrm{A} \mu_{\mathrm{n}} \prod_{\mathrm{j}=1}^{\mathrm{n}-1}\left(1-\mu_{\mathrm{j}}\right),
$$

где $\mathrm{m}_{\mathrm{n}}$ - единичная корреспонденция (транспортная единица)

А- объем отправления

$\mu_{\mathrm{n}}$ - вероятность, что посылка остановится в центре $\mathrm{n}$ при его достижении.

Данное выражение можно интерпретировать так: объем корреспонденции в центр системы прямо пропорционален произведению вероятностей остановки и вероятности того, что посылка не остановилась на пути ранее.

При расчете корреспонденций по сети условную вероятность остановки в центре определяют через емкость центра по прибытию. В случае многих центров передачи возникает проблема формального определения вариантов остановок на пути/на тракте. Чаще всего применяют градацию центров прибытия, т.е иерархию остановочных узлов по критерию удаленности от центра отправления. С такой позиции всю корреспонденцию пересылки можно представить выражением:

$$
M_{i j}=A_{i}\left(\exp \left(-\mu G_{j}\right)-\exp \left(-\mu G_{j+1}\right)\right),
$$

$\mu$-константа,

$\mathrm{G}_{\mathrm{j}}$ - емкость по прибытию всех центров, предшествующих центру $\mathrm{j}$.

\section{Выводы}

Таким образом, можно сделать следующие выводы:

1. Существуют 3 условные группы моделей сети передачи информации / транспортной сети: прогнозные, имитационные и оптимизационные.

2. В рамках наиболее востребованной из групп (имитационной) происходит подробное описание структуры, динамических и количественных характеристик сети через составление матрицы корреспонденций, элементы которой описывают объемы передвижений между условными районами/каналами.

3. Особенностью моделирования по такому направлению так же является соблюдение балансного подхода.

4. В работе описаны некоторые модели формирования матрицы корреспонденции (гравитационная, энтропийная, конкурирующих центров, промежуточных возможностей).

5. Их анализ, дает право утверждать, что :

- Гравитационная модель основана на расчете доступности центров прибытия и рассматривается изолированно от альтернативных центров.

- Модель промежуточных возможностей учитывает взаимное расположение альтернативных вариантов прибытия, но не принимает во внимание фактор доступности узла/канала.

- Недостаток гравитационной модели в части парной связности районов/каналов компенсирован в модели конкурирующих центров. 
- Энтропийная модель может служить статистическим обоснованием для гравитационной модели, имея возможность выбора функции тяготения.

6. Учитывая слабые стороны описанных выше моделей, исследователи работают над созданием комбинированных вариантов (например, гравитационноконкурирующая модель энтропийного типа), которые агрегируют достоинства базисных моделей, при этом стремятся минимизировать их недостатки.

7. Работоспособность той или иной модели явно определяется условиями, требованиями и особенностями конкретной практической задачи.

\section{References:}

1. Алиев А.С., Попков Ю.С., Швецов В.И. Моделирование транспорта в ИСА РАН // Компьютерные модели развития города. СПб.: Наука, 2003. С. 78-89.

2. Алиев А.С., Стрельников А.И., Швецов В.И., Шершевский Ю.З. Моделирование транспортных потоков в крупном городе с применением к Москвской агломерации. Автоматика и телемеханика, № 11, 2005. С.113-125.

3. Лившиц В.Н. Автоматизация планирования и управления транспортным и системами. М.: Транспорт, 1987. - 206 с.

4. Пакляченко М.Ю. Многоканальные информационные системы с параллелизмом каналов: характеристики и модели. / Исследования основных направлений технических и физико-математических наук: сборник научных трудов по материалам I Международной научной конференции 10 февраля 2014 г. Волгоград: Издательство «Научное обозрение», 2014. - С. 55 - 58.

5. Пакляченко М.Ю. Применение итерационных методов решения систем линейных алгебраических уравнений для анализа многоканальных систем обработки информации // Молодежный научно-исследовательский журнал. - 2013. №12 (19). C. $118-120$.

6. Попков Ю. С., Посохин М. В., Гутнов А. Э., Шмульян Б. Л. Системный анализ и проблемы развития городов. М.: Наука. Главная редакция физико-математической литературы, 1983. - 512 с.

7. Приходько М.А. Перераспределение информационных потоков в узлах распределенной мультисервисной системы обработки разнородной информации // Прикаспийский журнал: управление и высокие технологии. - 2012. № 1 (17). С 4752.

8. Стенбринк П.А. Оптимизация транспортных сетей // П. А. Стенбринк; пер. с англ. М.: Транспорт, 1981. - 320 с.

9. Швецов В.И. Математическое моделирование транспортных потоков. // АиТ. 2003. № 11. C. 3-46.

10. Wilson A. G. Entropy in urban and regional modelling. London: Pion, 1970. -845 p.

11. Stouffer S.A. Intervening opportunities: a theory relating mobility and distance // American Sociological Review. 1940. V. 5. P. 845-867. 